\title{
IMPACT OF DOPANTS ON THE CHARACTERISTICS OF THIN-FILM HUMIDITY SENSOR ELEMENTS
}

\author{
Nenov T. ${ }^{(1)}$, Kozhukharov S. ${ }^{(2)}$, Nenova Z. ${ }^{(1)}$, Machkova M. ${ }^{(2)}$ \\ ${ }^{(1)}$ Technical University of Gabrovo, 4 H.Dimitar Str., 5300 Gabrovo, Bulgaria \\ (2) University of Chemical Technology and Metallurgy, 8 Kliment Ohridski Blvd., 1756 Sofia, Bulgaria
}

\begin{abstract}
The paper presents the results from the study of thin-film humidity sensor elements on the basis of $\mathrm{TiO}_{2}$ obtained by a sol-gel method and doped with various compounds. The impact of the dopants on the sensor characteristics has been investigated. Titanium n-butoxide is used as a starting material for the preparation of the experimental samples. Bismuth acetate, vanadium pentadionate and sodium butoxide have been used as doping agents. The film is applied through the dip-coating method of the ceramic substrates in the obtained film-forming solution with subsequent drying in air environment. The dependence of resistance on humidity, sensitivity and response time of the sensor were investigated. Impedance characteristics and equivalent electric circuits were obtained.
\end{abstract}

\section{INTRODUCTION}

Humidity measurement in mixtures of gases - and in the air in particular - is a widely researched topic in a number of scientific and technical fields. Air humidity or the humidity of a technological gas is one of the important parameters, determining the quality of the products of a multiple of technological processes. Therefore it is important to improve on the already existing and develop new humidity sensors. One of the directions for addressing this is the use of new materials and technologies for preparing sensor elements. The humidity sensing elements can be prepared from oxide materials on the basis of ceramic technologies [1]. The benefit of using ceramic technologies for this comes from the fact that the microstructure of the ceramic materials could be controlled relatively easily through both the precursor's composition, and the synthesis conditions. In addition, the ceramic materials can be used for high temperature processes, and in aggressive media, because of its significant thermal and chemical durability. Lastly, the ceramic materials can be obtained via relatively simple operations.

According to specificities in their realization, humidity sensors can be divided into three types: thin-film, thick-film, and bulk. The last type of ceramic humidity sensors is prepared via application of a standard ceramic technology. The thick-film sensors are produced by deposition of appropriate paste on a substrate. The thin-film sensors are prepared via sol-gel based technologies.

In recent years there has been increased interest in the use of the sol-gel method for preparation of ceramic humidity sensors [2, 3]. That method enables the synthesis of nanostructured ceramic films. The specific features of the nanostructured materials should lead to humidity sensors with remarkably improved parameters and characteristics. This method leads to decrease in the size of the humidity sensors, as well as facilitates their integration in the semi-conductors technology.

The present paper presents the results from the study of thin-film humidity sensor elements on the basis of $\mathrm{TiO}_{2}$ obtained by a sol-gel method and doped with various compounds. The elements used as dopants are $\mathrm{V}, \mathrm{Bi}$ and $\mathrm{Na}$. The impact of the dopants on the sensor characteristics has been investigated.

\section{EXPERIMENTAL}

\subsection{Sample preparation}

The sol-gel colloidal system can be obtained by slow dripping of the corresponding metal alkoxides in a liquid medium, composed of alcohols, with the addition of water or acid as a catalyst. The basic processes related to the sol-gel technology are as follows: hydrolysis of metal alkoxides (reaction 1), polymerization of the hydrolyzed products (reaction 2), and partial evaporation of the liquid medium [4]

$$
\begin{aligned}
\mathrm{Ti}\left(\mathrm{OC}_{4} \mathrm{H}_{9}\right)_{4}+4 \mathrm{H}_{2} \mathrm{O} & \rightarrow \mathrm{Ti}(\mathrm{OH})_{4}+4 \mathrm{C}_{4} \mathrm{H}_{9} \mathrm{OH} \\
\mathrm{Ti}(\mathrm{OH})_{4} & \rightarrow \mathrm{TiO}_{2}+4 \mathrm{H}_{2} \mathrm{O} \uparrow .
\end{aligned}
$$

All of the procedures for preparation of products via the sol-gel method are based on the same sequence, represented by equations (1) and (2).

In practice, there is a variety factors that predetermine the quality of the corresponding products [5]. As 
with all other chemical technologies, the conditions for synthesis entirely predetermine the structure, and consequently, the properties of the respective product. Some of the most important factors are the nature and content of the precursors, presence of solvents, influence of $\mathrm{pH}$ of the medium, temperature, influence of the surrounding gaseous environment, etc.

Titanium n-butoxide (TBOT) was used as a starting material for the preparation of the experimental samples, and the solvent was 1-buthanol. Nitric acid was added as a catalyst of the hydrolysis process. After the exposure of the solution at $70^{\circ} \mathrm{C}$ for 30 minutes, in a closed vessel the solution was used for film depositions. That procedure was performed via the dip-coating method with subsequent drying in air environment [4]. The substrates were corundum $\left(\mathrm{Al}_{2} \mathrm{O}_{3}\right)$ plates with previously deposited interdigitated silver-paladium electrodes.

In order to investigate the influence of various dopants on the characteristics of the sensors, three different initial solutions were prepared. After mixing of the buthanol with the TBOT, the obtained solution was heated to $70^{\circ} \mathrm{C}$. Afterwards, there solutions were made by adding a particular dopant to each. Specifically, the first one includes Vanadium (III)-2,4 pentadionate, the second includes Bismuth acetate, and the third includes Sodium tert-butoxide. After addition of the catalyst $\mathrm{HNO}_{3}$, the obtained sols were thermally treated at $70^{\circ} \mathrm{C}$ in a thermostat (in covered beakers).

The substrates were dipped in the sol three times for 20 minutes and were allowed to dry between dippings. After preparation and drying of the samples they were sintered at $400^{\circ} \mathrm{C}$ for 30 minutes to obtain a ceramic thin film. The experimental samples were named, as follows: TV - doped by Vanadium(III)-2,4 pentadionate, TB - Bismuth acetate, and TN - Sodium tert-butoxide.

Figure 1 represents the experimental samples obtained after the application of the process described above.

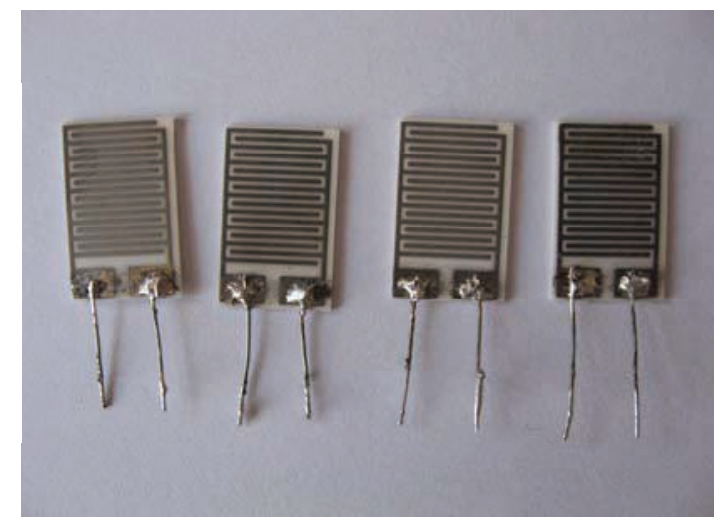

Figure 1. Thin film samples of humidity sensing elements

\subsection{Measurement of the sample parameters}

The humidity sensing characteristics of the samples were determined in conditions of controlled humidity through a relative humidity calibrator VAPORTRON H-100BL, product of BUCK RESEARCH INSTRUMENTS L.L.C. [6]. The device supplies values of relative humidity in the range from 10 to $95 \%$, with precision of $1.5 \%$. The active resistance and the impedance of the sensors, in the conditions of the humidity chamber were measured by an impedance analyzer Precision Impedance Analyzers 6505P product of Wayne Kerr Electronics Ltd. at a frequency of $1 \mathrm{kHz}$ and amplitude of $500 \mathrm{mV}$ of the testing signal. The impedance analyzer permits evaluation of various parameters such as, phase angle, capacitance, resistance, inductance, and quality factor with a basic accuracy of 0,05\% [7].

\section{RESULTS AND DISCUSSION}

Figure 2 illustrates the functional dependence of the resistance $R=f(R H)$, and the phase angle $\theta=f(R H)$ on relative humidity $\mathrm{RH}$ for the sample TV, doped by Vanadium(III)-2,4 pentadionate.

The sample TV has good sensitivity in the entire range of relative humidity from $15 \%$ to $95 \%$. This sensitivity decreases as $\mathrm{RH}$ increases at a rate of $2.2 \mathrm{M} \Omega / \% \mathrm{RH}$, for the initial interval of 15 to $30 \% \mathrm{RH}$, up to $930 \mathrm{k} \Omega / \% \mathrm{RH}$ for the final interval part of the humidity range (i.e. 75 to $95 \% \mathrm{RH}$ ). In addition, the phase angle changes by about $20^{\circ}$ for the entire range of $\mathrm{RH}$ (average sensitivity $0.3^{\circ} / 1 \% \mathrm{RH}$ ). 


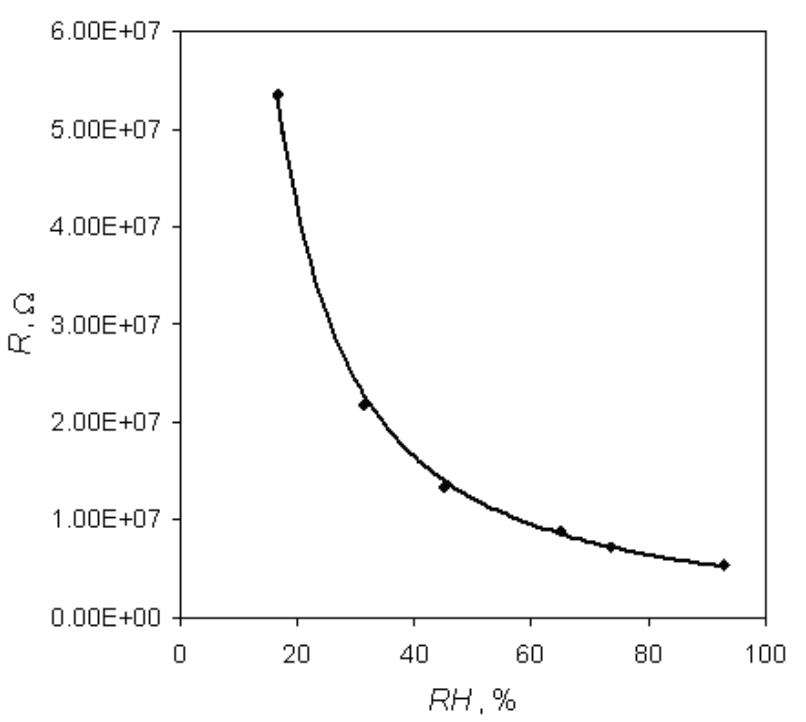

a

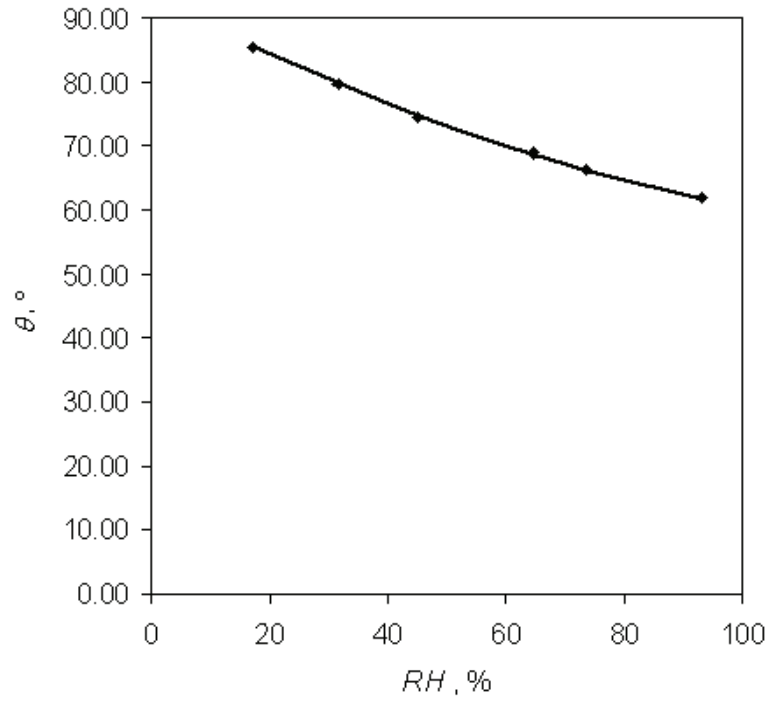

$\mathrm{b}$

Figure 2. Dependence of resistance (a) and phase angle (b) on RH for sample TV

Good sensitivity in the range $30 \%$ to $95 \% \mathrm{RH}$ is revealed by the sample TN, doped with Sodium tertbutoxide. It is observed that the sensitivity decreased at lower RH (see Figure 3). It undergoes changes, beginning from $151 \mathrm{k} \Omega / \% \mathrm{RH}$ for the initial interval (15 to $30 \% \mathrm{RH}$ ) to $1.3 \mathrm{M} \Omega / \% \mathrm{RH}$ for the final interval (65 to $73 \% \mathrm{RH}$ ). In that sample, a significant change in the phase angle, with more than $75 \%$ of $\mathrm{RH}$ is registered. The phase angle is $65^{\circ}$ for the interval $75-95 \% \mathrm{RH}$, which is equal to $3.4 \% \mathrm{RH}$ sensitivity.

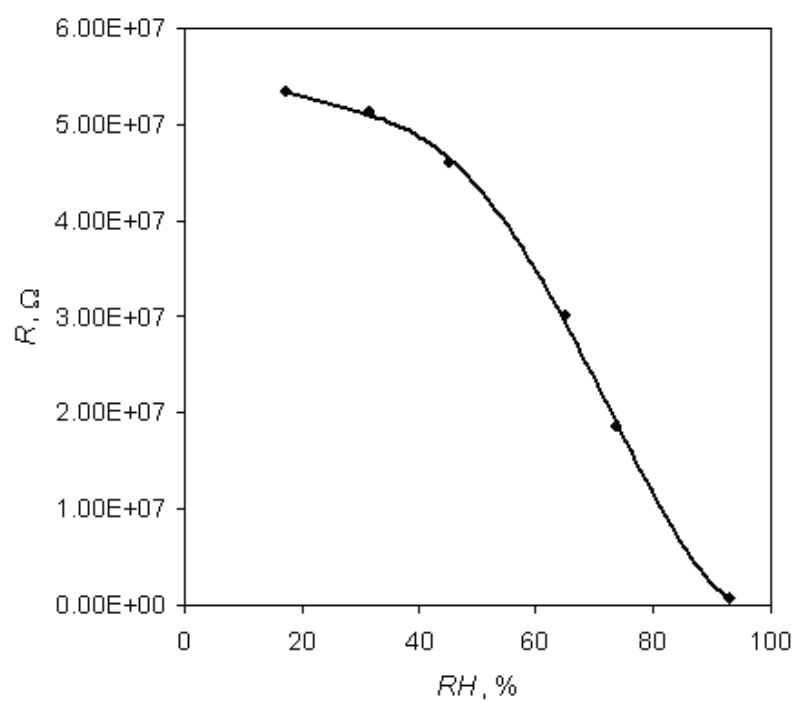

a

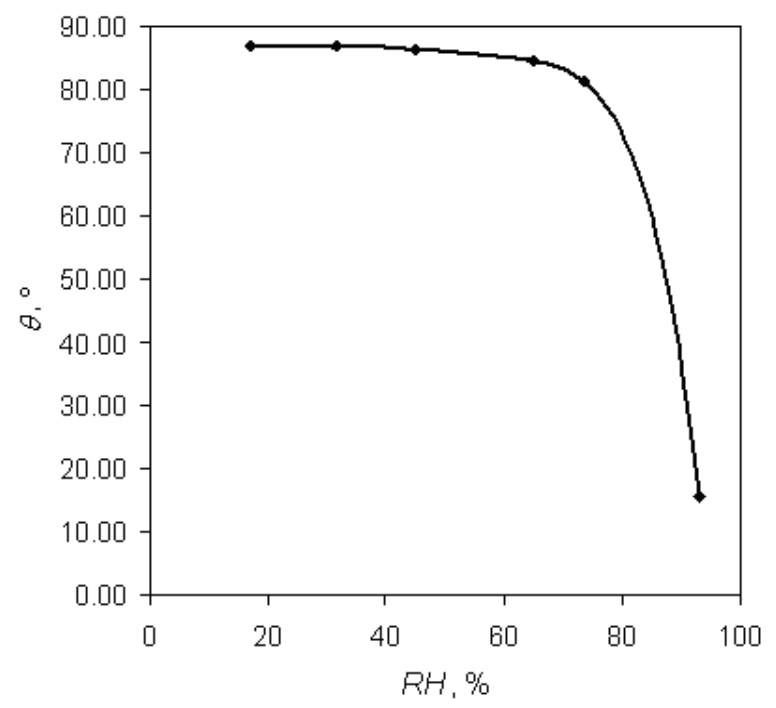

$\mathrm{b}$

Figure 3. Dependence of resistance (a) and phase angle (b) on RH for sample TN

Figure 4 shows the characteristic of the sample TB, doped with Bismuth acetate. That sample reveals sensitivity only at values of $\mathrm{RH}$, higher than $65 \%$. This sensitivity reaches $2.9 \mathrm{M} \Omega / \% \mathrm{RH}$ for the interval $75-95 \% \mathrm{RH}$. Only a negligible change in the phase angle is registered at values above $65 \%$.

The results obtained lead to the conclusion that doping with the Bi-compound does not influence significantly the humidity sensitivity of the respective thin-film ceramic sensors, compared to similar samples, based on $\mathrm{TiO}_{2}[8]$. 


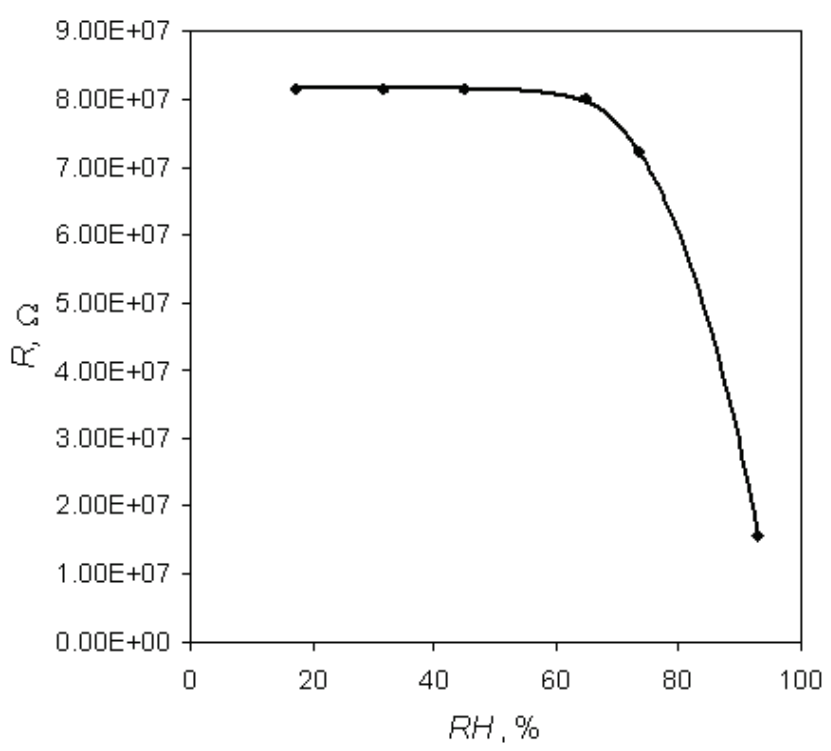

a

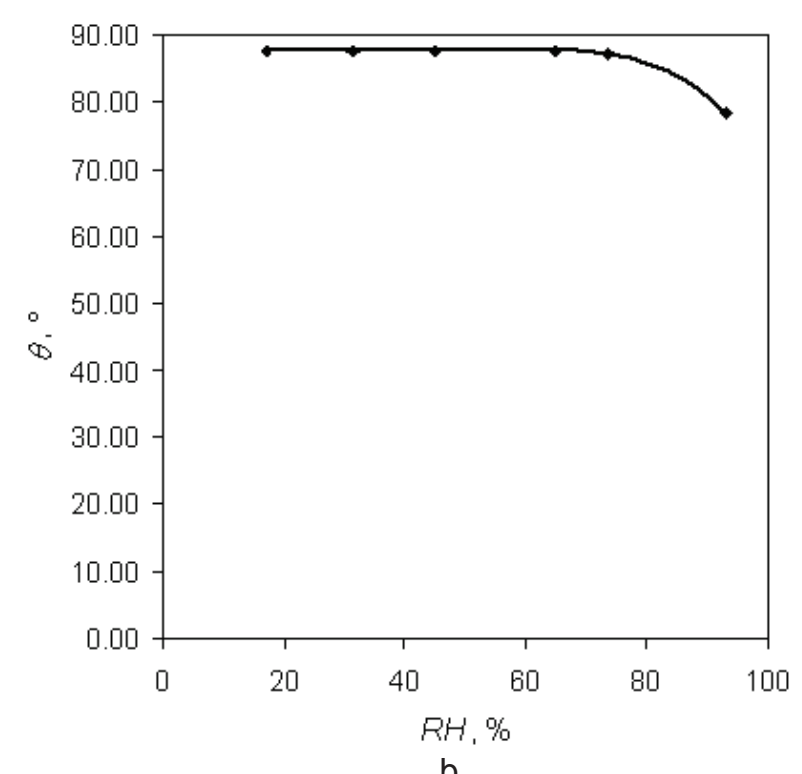

b

Figure 4. Dependence of resistance (a) and phase angle (b) on $\mathrm{RH}$ for sample $T B$

The change of the resistance of the respective sensors at adsorption/desorption, as well as the influence of the doping components over the reaction delay is also assessed. Figure 5 represents the change in resistance for the sample TN. It is characterized by the lowest reaction delay for changes in $\mathrm{RH}$. The response time for one cycle of adsorption/desorption was less than 20 seconds.

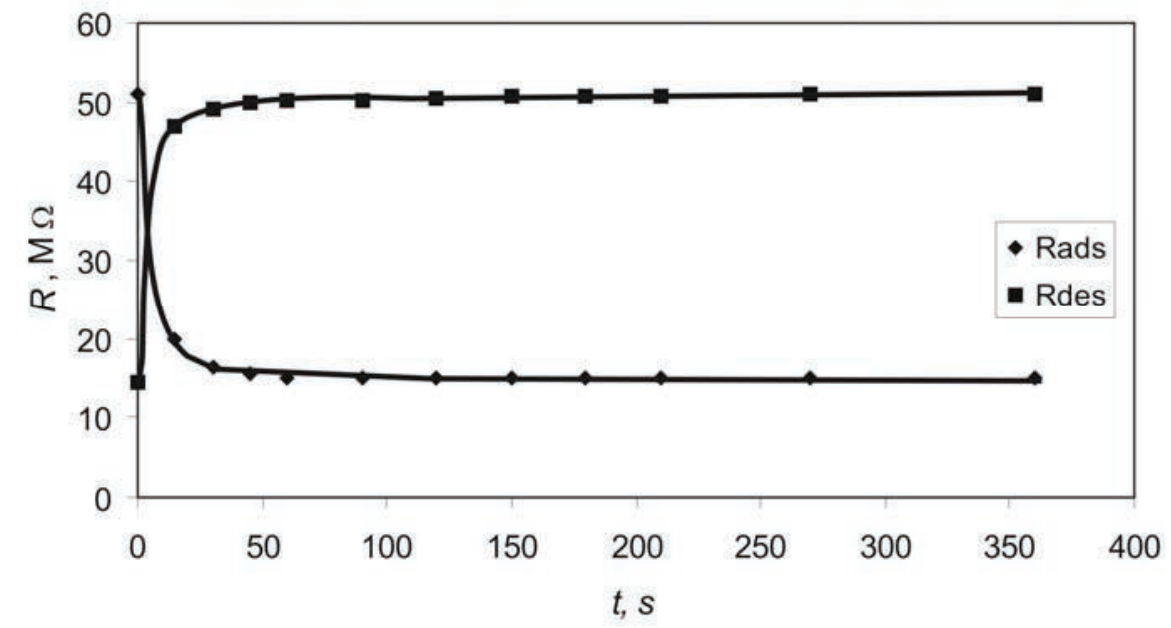

Figure 5. Change in resistance during adsorption and desorption for sample TN

The response time for the sample TV at adsorption was about 300 seconds, while at the subsequent desorption, it was larger than 600 seconds. The response time for the sample TB at desorption was lower than at adsorption. It was about 270 seconds at adsorption, and around 120 for desorption, respectively. Thus, the samples doped by the Na-substance revealed the lowest response time to changes in humidity. The hysteresis in the characteristics of the sensor elements was investigated as well. It is largest for sample TV and relatively low for samples TN and TB.

The impedance characteristics of the samples were also investigated. These characteristics were used for the creation of the $\mathrm{Xc}(\mathrm{R})$ diagrams (Figure 6$)$ and the equivalent electric circuits of the samples. They consist of a resistor and capacitor connected in parallel, with values determined by the $\mathrm{Xc}(\mathrm{R})$ diagram. 


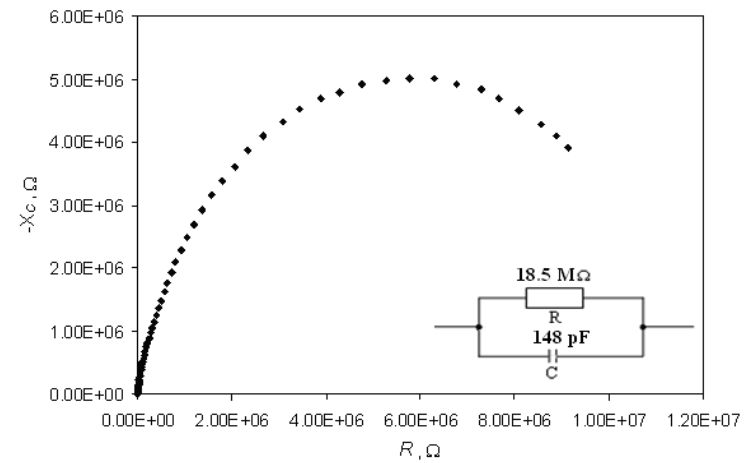

a

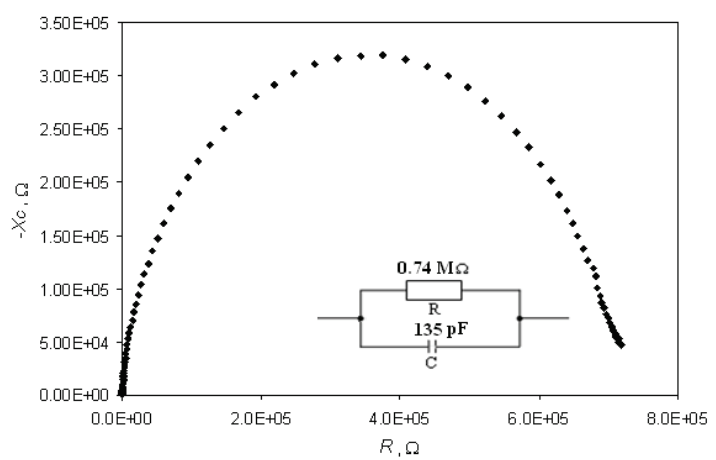

$\mathrm{b}$

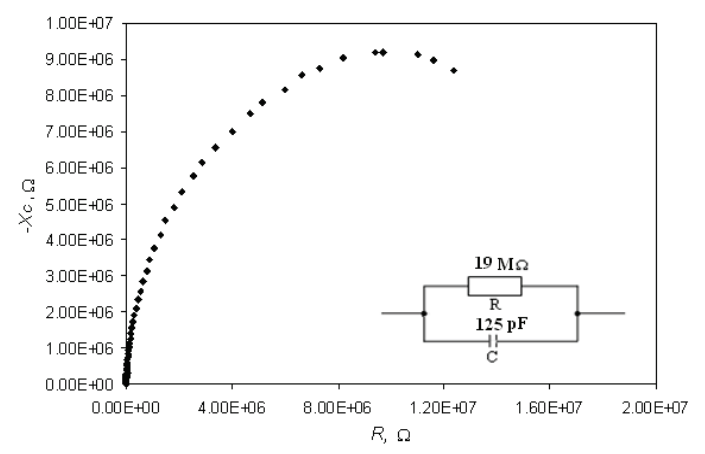

C

Figure 6. $X c(R)$ diagrams and equivalent electric circuits of experimental samples:

a) $T V$, b) $T N$ and c) $T B$

\section{CONCLUSION}

Based on the above investigation of the effect of dopants on the characteristics and parameters of the sensing elements the following conclusions can be made:

- doping of the titanium dioxide based films influences the sensitivity of the humidity sensing elements;

- the different doping compounds used for modifying the sol composition impact differently the sensitivity and the range of relative humidity, in which it is expressed. A substantial increase in sensitivity and an expansion of the measurement range is obtained with the doping of Vanadium (III)-2,4 pentadionate and Sodium tert-butoxide;

- sodium doped samples also have good sensitivity in the 30\%-50\%RH range;

- doping with bismuth does not significantly influence the humidity sensitivity of the elements obtained but they can find applications as key humidity sensing elements;

- the sodium doped samples have the shortest reaction time to changes in humidity;

- the characteristics of samples doped with sodium and bismuth have relatively low hysteresis.

\section{ACKNOWLEDGEMENT}

The results presented here are in fulfillment of contract № ДО 02-148/2008г. financed by the National Scientific Research Fund (Bulgaria).

\section{REFERENCES}

[1] Nenov, T., S.Yordanov. Ceramic Sensors: Technology and Applications. TECHNOMIC Publ. Co., Inc.Lancaster-Basel, 1996.

[2] Biju K. P., M. K. Jain. Effect of crystallization on humidity sensing properties of sol-gel derived nanocrystalline $\mathrm{TiO}_{2}$ thin films. Thin Solid Films, Vol. 516, 2008, pp.2175-2180. 
[3] Erol A., S.Okur, B.Comba, O.Mermer, M.C.Arikan. Humidity sensing properties of ZnO nanoparticles synthesized by sol-gel process. Sensors and Actuators B:Chemical, 2010, Vol.145, pp.174-180.

[4] Brinker C.J., G.W.Scherer. Sol-Gel Science. The Physical and Chemistry of Sol-Gel Processing. Academic Press. San Diego-New York-Boston, 1990.

[5] Petrov G. Organic chemistry. KI. Okhridsky Publ., Sofia, 1996.

[6] VAPORTRON H100BL/H100CL Series Precision Humidity Lab. Buck Research Instruments L.L.C., Boulder CO, 2009. (http://hygrometers.com/wp-content/uploads/VAPORTRON-usersmanual-2009-12.pdf).

[7] HF LCR Meter 6505. Technical data sheet. Wayne Kerr Electronics Ltd., 2008. (http://www.waynekerrtest.com/brochure/6500P\%20Series.pdf)

[8] Nenova, Z., T. Nenov, S. Kozhukharov. Thin Film Humidity Sensing Elements. International Scienetific Conference UNITECH'10, 19 - 20 November 2010, Gabrovo. Proceedings, Vol. I, p. I$512-1-517$. 